\title{
Strengthening the case for goal directed fluid therapy
}

\begin{abstract}
Intravenous (IV) fluids are critica for maintaining hydration and blood circulation during major abdominal surgery. Th right amount of fluids can help prevent complications recovery, but finding that swe spot can be challenging. To help narrow the field, Beijing Tongren Hospital Department of Anesthesiology Vice-Chief Anesthesiologist, Dr Yanxia Sun and her team are running a clinical trial, testing the effectiveness of a treatment guided fluid therapy'.
\end{abstract}

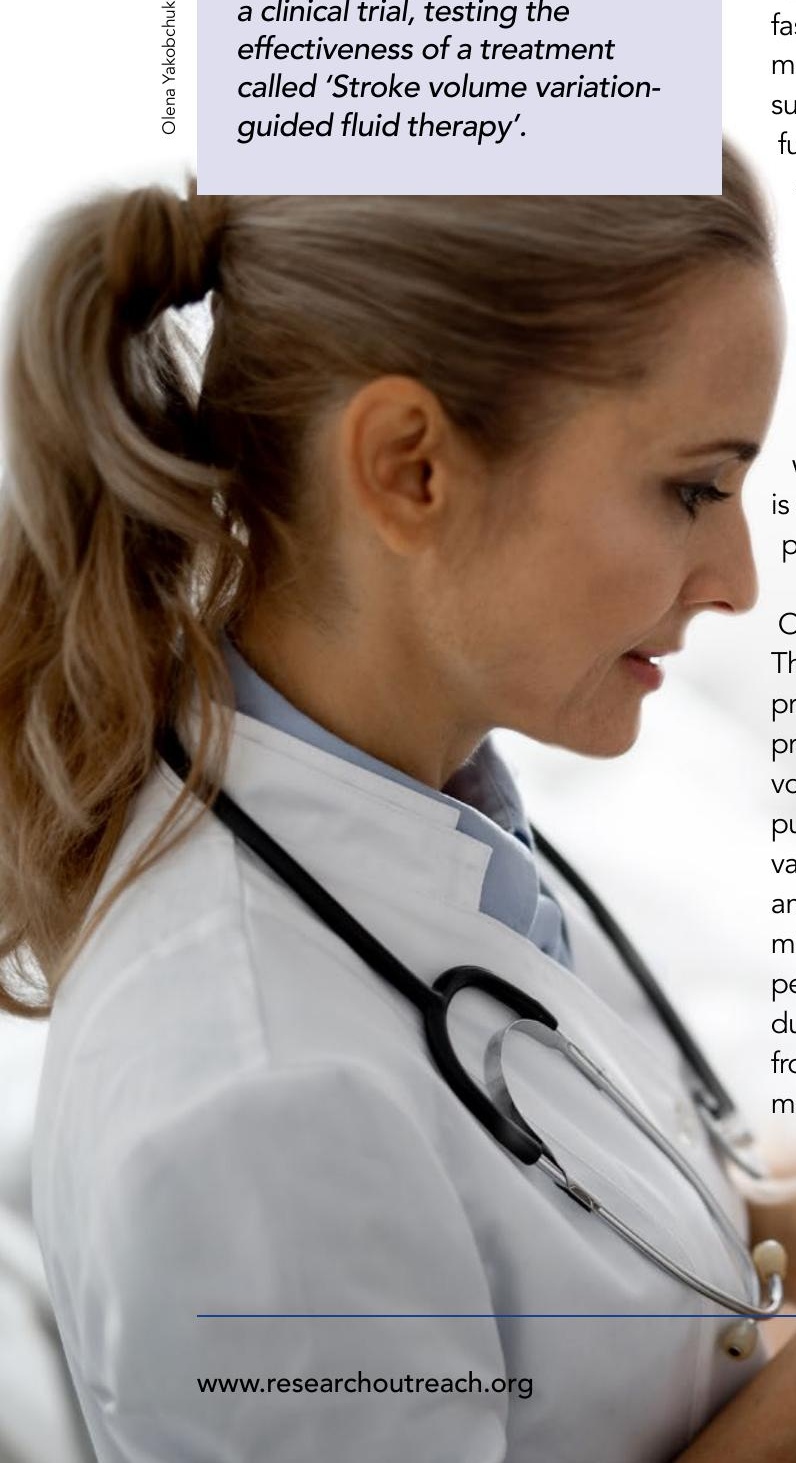

ntravenous (IV) fluids - or drips - a ubiquitous in hospitals and often used to treat dehydration, infection, cancer and pain. They're also used during surgery to maintain hydration not only ensures a patient's tissues enough blood, water electrolytes and oxygen while they're under the knife, but has also been shown to affect postoperative recovery - particularly following major abdominal surgery. Given enough fluids, patients can have a better chance at recovering faster without any complications. Too much or too little and the patient can suffer organ dystunction, delayed gut function recovery and increased risk of complications after surgery.

Yet, achieving optimal fluid management can be challenging particularly using parameters such venous ressure or ressue, central which poorly estimate how much flu is reaching the heart and how much itw pump out.

One solution is 'Goal Directed Fluid Therapy' (GDFT). Here, medical practitioners administer fluid based on a predetermined goal, such as stroke volume - the amount of blood a heart pumps out in one beat, stroke volum variation (SW), cardiac output - the amount of blood pumped out in one pexygen delivery. $\mathrm{S} W$, as a entinge change of stroke volume from its relationship with the ( minimum stroke volume, and mean stroke volume over a 20-second time interval and expressed as follows: $\mathrm{SW}(\%)=$ stroke volume/mon strokino volume.

This practice has been steadily gaining popularity, but despite numerous studies, researchers still haven't reached a consensus on which measurement is best or how effective GDFT really is.

To help determine the true value of SW-based GDFT in abdominal surgery patients, Beijing Tongren Hospital Department of Anesthesiology Vice-Chie Anesthesiologist, Dr Yanxia Sun and her team are running a clinical trial, exploring participants' responses to the treatment and their shor and long term prognoses.

TAKING A PULSE ON GDFT Improving patient outcomes is a major goal of modern medicine and one that been trying to achieve through fluid therapy for years. But conflicting study results have prevented researchers from identifying the best GDFT goals or monitoring devices for the job. Indeed, the effectiveness of GDFT has been inconclusive despite increasing clinica trials and meta-analyses.

Where some studies have shown GDF to help improve survival rates, reduce complications and support gut function recovery in major abdominal surgery patients, larger, higher-quality studies have
failed to replicate these beneficial effects.

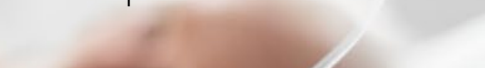

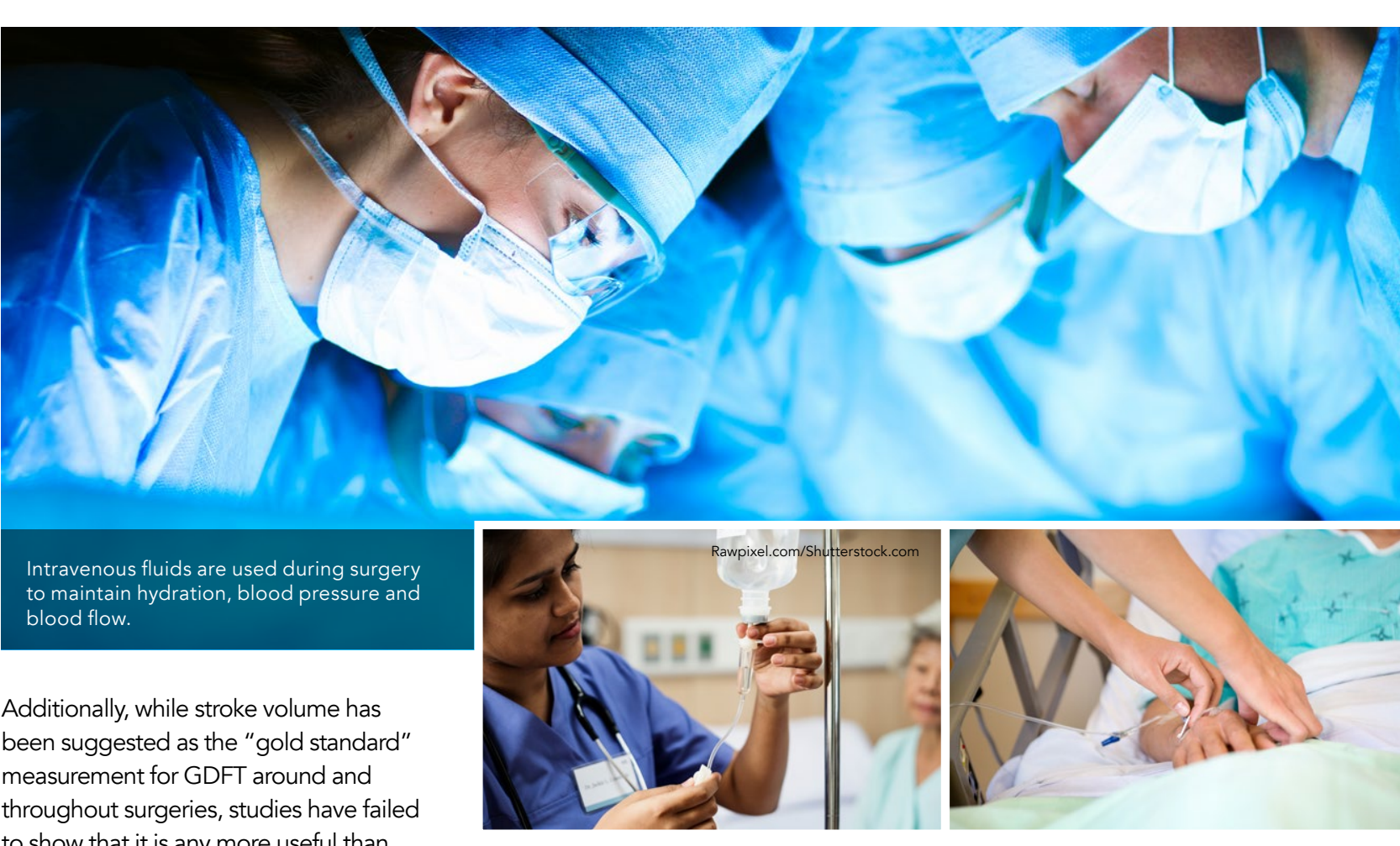

troughout surgeries, studies have failed

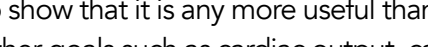
index - which measures the amount of blood pumped around the body per minute, or oxygen delivery index.

Further, researchers haven't nailed down the most appropriate type of monitoring device, with oesophageal doppler, calibrated pulse contour analyses and bioreactance-based non-invasive cardiac output monitors all being used and very ittle agreement between them

Variation in surgical methods and study approaches, sizes and data quantity and quality have all cont medical practitioners from optimising patient care.

As a result, researchers have questioned whether GDFT's "routine" use is justifiable, given it can increase costs, lead to inappropriate patient care and has been shown to have minimal - if any - benefits when used as part of an 'enhanced recovery after surgery' (ERAS) programme. These programs are designed to improve patient outcomes, reduce healthcare costs and shorten recovery time by using minimally invasive surgeries such as laproscopies, avoiding overloading patients with huids or using surgery limiting the use of opoids in pain surgery, limiting the use of opioids in pain

Given enough fluids, patients have a better complications.

drains and catheters, among other methods. Many of these elements can help minimise bodily stress and blood loss Achieving optimal
can be challenging

In a letter to Anesthesia and Analgesia, Cannesson and Gan also pose a more

.. medical practitioners hope to tease out and standardise best practices for fluid management and enhance patient care.

fluid requirements and GDFT's value during surgery.

However, other researchers argue that having fluid mangement goals - even if they're not solidly backed - is still better than not having one at all, particularly when it comes to patients who are moderate- to high-risk, undergoing major surgeries or outside an ERAS programme. Here, the researchers cite studies that have shown GDFT to help shorten

hospital stays, reduce complications an suggest that increased costs could be mitigated by working to minimise pulse pressure vantation rather than use devices like a cardlac output monitor. They add that, since institutions have standardised pain management protocols despite that GDFT protocols should be too. therapy or GDFT if they were having highrisk surgery tomorrow.

Either way, researchers and medica practitioners hope to tease out and standardise best practices for fluid care. This is where Dr Sun and her team come in...

STABILISING GDFT Knowing the contention around GDFT and having completed two meta-analyses on the topic herself, Dr Sun and her team designed a clinical trial to measure the effectiveness of SW-guided GDFT. During the trial, 100 adult major abdomin SW-ruided GDFT or con received therapy bed oD or conventinal fluid therapy based on central venous pressure, 


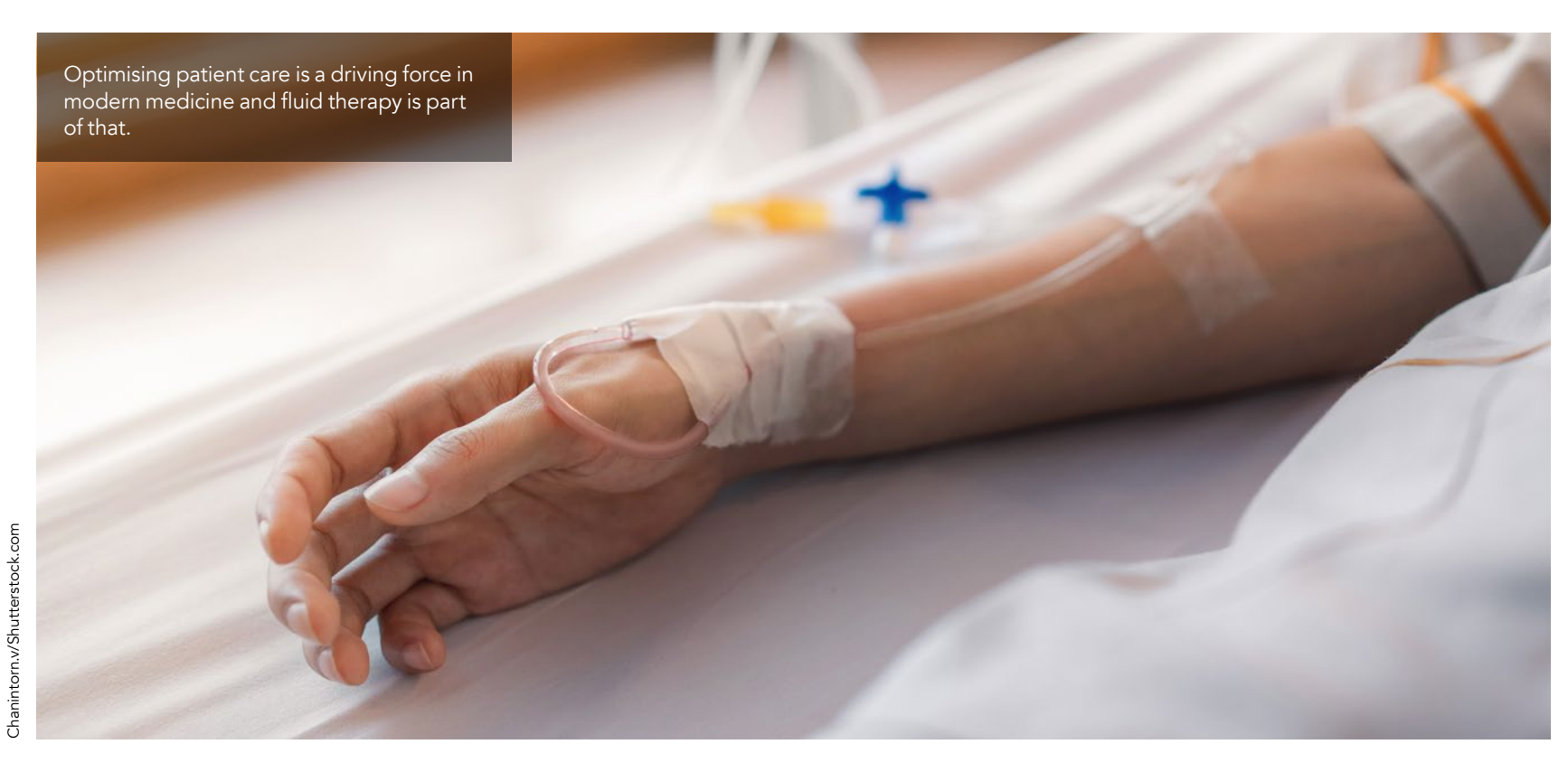

Here, researchers recorded patients' basic vital signs, operation time, mechanical urine volume, blood loss volume and the amount of oxygen consumed versus oxygen delivered.

In the year following the surgeries, the researchers will be checking in with the patients and recording whether there were any postoperative deaths or complications, hospital stay dur
quality of recovery and comfort.

The researchers hope this trial - set to be completed in August 2020 - will provide a clearer picture of SW-guided GDFT's
effect on long term prognoses in patients effect on long term prognoses in patien
undergoing abdominal surgery and undergoing abdominal surgery and
support its use in clinical settings.

IMPROVING PATIENT CARE Optimising patient care is a driving force is part of that Getting it right can help patients recover faster, spend less time (a) parameters such as heart rate, blood or urine output, but these fail to

...Dr Sun and her team hope to help strengthen the case for clinical use of SVV-guided GDFT...

in hospital and avoid complications adequately estimate how much fluid during or after surgery, but getting it is reaching or being pumped out wrong can expose them to increased of the heart - making it difficult to risk of complications, organ dysfunction, maintain patients' hydration, blood longer recovery times and hospital stays pressure and blood flow. and higher costs. Therefore, it's critical for researchers to isolate and standardise GDFT has been offered as a solution, however, even with mounting studies, however, even with mounting studies,
researchers have struggled to confirm its effectiveness and which parameters and machinery are best for the job. As a result, researchers are calling for more, higherquality studies to help determine whether GDFT really is beneficial to patients and under which circumstances. Ideally, they hope to determine best practices for fluid therapy and standardise them to enhance patient care.

Through their clinical trial, Dr Sun and her team hope to help strengthen the case for clinical use of SW-guided GDFT and avoid the pittalls of previous studies. With any luck, by August next year, the group will have the data and means to do just that and bring researchers and medical practitioners
fluid therapy.

\section{Behind the Research in \\ DrYanxia (Sunny) Sun

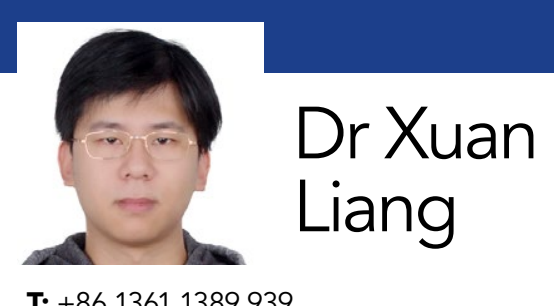 \\ E: sun00017@gmail.com T: +861361 1389939}

\section{Research Objectives}

Dr Yanxia (Sunny) Sun's research aims to explore the effect of goal-directed fluid therapy on postoperative outcome and long-term prognosis of patients undergoing abdominal surgery, with a view to reducing postoperative complications, shortening hospital stay and improving longterm prognosis

\section{Detail}

Department of Anesthesiology

Beijing Tong Ren Hospital
1 Dong Jiao Min Xiang Street

Beijing 100730 China

Bio

DrYanxia Sun M.D. Ph.D. was Research Fellow for clinical research at the Department of Anesthesiology, Duke Medical Center, Durham, NC, USA between 2006 and 2008. She was Vice Director for Medical Research Center at Beijing TongRen Hospital between 2012 and 2016. Dr Sun is currently the Vice-Chief Anesthesiologist within the Department of Anesthesiology Bejing Tongren Hospital, Capital Medical University, Beijing.

Funding

- Research Development Program of Beijing

Hospital Authority

Collaborators
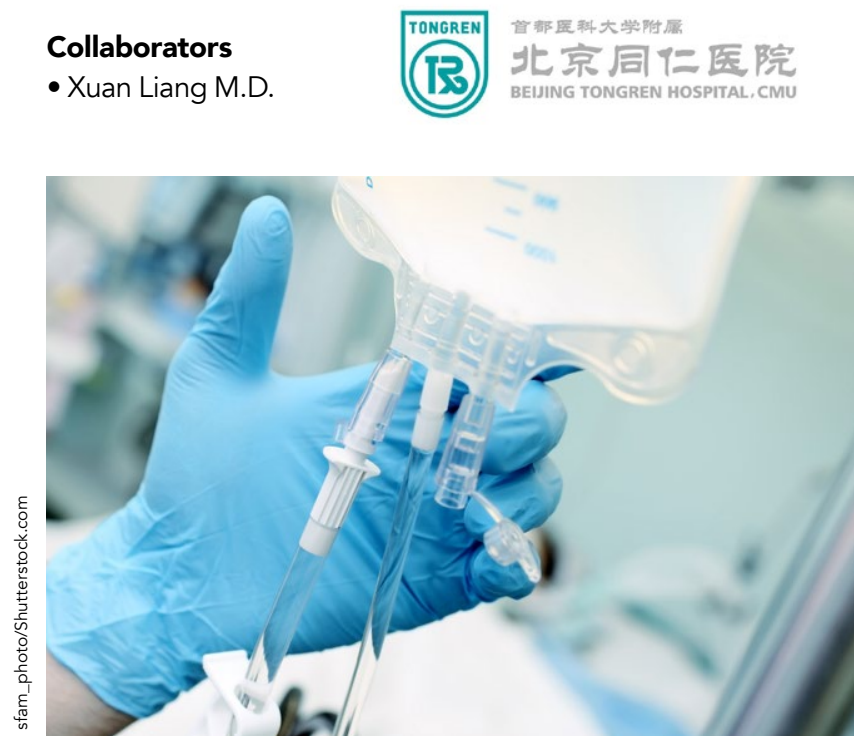

References

Sun, Y., Chai, F., Pan, C., Romeiser, J. and Gan, T. (2017). Effect of perioperative goal-directed hemodynamic therapy on postoperative recovery following major abdominal surgery-a systematic review and metaanalysis of random s13054-017-1728-8.

Yuan, J., Sun, Y., Pan, C. and Li, T. (2017). Goal-directed fluid therapy for reducing risk of surgical site infections following of randomized controlled trials. International Journal of Surgery, [online] 39, 74-87. doi:10.1016/;.jisu.2017.01.08 (2019). Effect of SW-guided Fluid Therapy on Outcomes After Major Abdominal Surgery. [online] ClinicalTrials.gov. Available at: https://clinicaltrials.gov/ct2/show/NCT03940144

\section{Personal Response}

What have you learned from other studies that have gthen the value of this trial, compared to others?

II Stroke volume variation (SVV)-GDFT, is attracting

increasing attention as a reliable, less invasive and easy to use strategy for protocolised fluid management. There have SVV-GDFT on postoperative recovery after major surgery, for postoperative recovery after abdominal surgery remains evaluating fluid responsiveness was not clear, therefore we have performed this large-size study to evaluate the efficacy also aim to identify the cut-off SVV value for perioperative fluid therapy for major abdominal surgery. With so much confusion about which parameters are II It has been suggested that intraoperative fluid administration may be better guided by flow related parameters. Stroke volume variation (SVV) is an accurate parameter which is influenced by cardiopulmonary mechanical ventlation, an acurately predict whether a patient is likely to be responsive or nonresponsive to volume expansion and indicates whether the patient is on the steep portion or on the plateau of the Franck Starling relationship. Consequently, the concept of cardiac output maximisation. we chose this parameter to guide our GDFT protocols. 